\title{
ALERGIA A FÁRMACOS COM MANIFESTAÇÕES CUTÂNEAS - ABORDAGEM DIAGNÓSTICA
}

\author{
João Antunes', Susana Brás², Sara Prates ${ }^{3}$, Cristina Amaro ${ }^{4}$, Paula Leiria-Pinto 5 \\ 'Interno de Imunoalergologia/Resident Immunoallergology, Serviço de Imunoalergologia/Department of \\ Immunoallergology, Hospital de Dona Estefânia - Centro Hospitalar de Lisboa Central \\ Interna de Dermatologia e Venereologia/Resident Dermatology and Venereology, Serviço de Dermatologia e \\ Venereologia/Department of Dermatology and Venereology, Hospital de Curry Cabral - Centro Hospitalar de Lisboa \\ Central \\ ${ }^{3}$ Assistente Hospitalar de Imunoalergologia/Consultant Immunoallergology, Serviço de Imunoalergologia/Department \\ of Immunoallergology, Hospital de Dona Estefânia - Centro Hospitalar de Lisboa Central \\ ${ }^{4}$ Assistente Hospitalar de Dermatologia e Venereologia/Consultant Dermatology and Venereology, Serviço de \\ Dermatologia e Venereologia/Department of Dermatology and Venereology, Hospital de Curry Cabral - Centro \\ Hospitalar de Lisboa Central \\ ${ }^{5}$ Directora do Serviço de Imunoalergologia/Head of the Department of Immunoallergology, Hospital de Dona \\ Estefânia - Centro Hospitalar de Lisboa Central
}

RESUMO - As reacções adversas a fármacos (RAF) representam um problema frequente na prática clínica. A alergia a fármacos resulta de mecanismos de hipersensibilidade imunológica e representa $6-10 \%$ do total de RAF. Clinicamente, as reacções alérgicas a fármacos podem ser classificadas como imediatas (tipo I) ou não-imediatas (com manifestações clínicas diversas e associadas sobretudo a reacções de tipo IV). Neste artigo são abordados aspectos gerais, nomeadamente os mecanismos imunopatogénicos implicados na alergia a fármacos e reactividade cruzada mas também as manifestações cutâneas mais relevantes, nomeadamente exantemas máculo-papulares, eritema fixo a fármacos (EFF), pustulose exantemática aguda generalizada (PEAG), síndrome de hipersensibilidade a fármacos (DRESS - drug rash with eosinophilia and systemic symptoms), síndrome de Stevens-Johnson/necrólise epidérmica tóxica (SSJ/NET). O papel dos testes cutâneos (epicutâneos ou intradérmicos de leitura tardia) na abordagem de reacções não-imediatas é também revisto. Os beta-lactâmicos (BL) são o grupo farmacológico mais frequentemente envolvido em reacções de hipersensibilidade imunológica e que mais dificuldades coloca na prática clínica diária, nomeadamente devido aos riscos de reactividade cruzada, pelo que é analisado em maior detalhe ao longo da revisão. A indução de tolerância a fármacos poderá ser considerada em casos selecionados, sobretudo quando na ausência de alternativas terapêuticas igualmente eficazes ou seguras.

PALAVRAS-CHAVE - Hipersensibilidade a fármacos; Beta-Lactâmicos; Reactividade cruzada; Testes cutâneos.

\section{DRUG ALLERGY WITH CUTANEOUS MANIFESTATIONS - DIAGNOSTIC APPROACH}

ABSTRACT - Adverse drug reactions constitute a major health problem in clinical practice. Drug allergy is defined as an immunologically mediated hypersensitivity reaction and represents $6-10 \%$ of all adverse drug reactions. Clinically, drug-induced allergic reactions can be classified as immediate (type I) and non-immediate (heterogeneous clinical manifestations, mostly related to type IV reactions). Several issues are considered in this revision, particularly immunopathologic mechanisms, cross-reactivity and the most relevant cutaneous reactions (maculopapular exanthema, fixed drug eruption, acute generalized exanthematic pustulosis, drug hypersensitivity syndrome, also referred as drug rash with eosinophilia and systemic symptoms - DRESS, Stevens-Johnson syndrome and toxic epidermal necrolysis). The role of skin tests (epicutaneous or intradermal with late reading) in the diagnostic approach of non-immediate reactions is also analysed. The most important causes of hypersensitivity reactions are antibiotics, particularly beta-lactams. This group poses significant diagnostic and management difficulties and deserves, therefore, a 


\title{
Educação Médica Contínua
}

more thorough attention, with particular attention to cross-reactivity patterns. Induction of drug tolerance should be considered in selected cases, especially when no safe or effective drug alternatives are available.

KEY-WORDS - Drug hypersensitivity; Beta-Lactams; Cross reactions; Skin Tests.

\section{Conflitos de interesse: Os autores declaram não possuir conflitos de interesse. \\ No conflicts of interest. \\ Suporte financeiro: O presente trabalho não foi suportado por nenhum subsídio ou bolsa. \\ No sponsorship or scholarship granted.}

Recebido/Received - Junho/June 2012; Aceite/Accepted - Julho/July 2012.

Por decisão dos autores, este artigo não foi redigido de acordo com os termos do novo Acordo Ortográfico.

\author{
Correspondêncio \\ Dr. João Antunes \\ Serviço de Imunoalergologia \\ Hospital de Dona Estefânia \\ Rua Jacinta Marto \\ $1169-045$ Lisboa \\ Tel.: + 351213126666 \\ Fax: +351213126667
}

Centro Hospitalar de Lisboa Central

\section{INTRODUÇÃO}

As reacções adversas a fármacos (RAF) representam um problema frequente na prática clínica, atingindo 10 a $20 \%$ dos doentes internados e $7 \%$ da população geral, e constituem uma importante causa de mortalidade intra-hospitalar ${ }^{1}$.

As RAF podem ser classificadas em reacções do tipo A, ("augmented") previsíveis, comuns (80\%), dependentes de dose e relacionadas com os mecanismos de acção dos fármacos e reacções do tipo B ("bizarre"), imprevisíveis, mais raras (20\%), independentes de dose e geralmente sem relação com a acção farmacológica. As reacções do tipo A subdividem-se em efeitos secundários do fármaco, quadros de toxicidade e interacções farmacológicas; as reacções do tipo $B$ englobam fenómenos de intolerância (resposta alterada com doses terapêuticas ou subterapêuticas, sem alterações do metabolismo, excreção ou biodisponibilidade do fármaco, ie. tinitus induzido pela aspirina), reacções de idiossincrasia (não explicadas pelos princípios farmacológicos e geralmente associadas a erros do metabolismo, excreção ou biodisponibilidade do fármaco, ie. anemia hemolítica induzida pela primaquina em doentes com défice de glucose-6-fosfato desidrogenase), reacções pseudoalérgicas (com estimulação directa e consequente desgranulação de mastócitos e basófilos, sem reconhecimento imunológico prévio, ie. opióides, vancomicina, colóides ou contrastes radiológicos) e reacções alérgicas. A alergia a fármacos resulta de mecanismos de hipersensibilidade imunológica e representa $6-10 \%$ do total de $\mathrm{RAF}^{2-4}$.

\section{REACÇÕES DE HIPERSENSIBILIDADE MEDIA- DAS PELO SISTEMA IMUNE (TABELA 1)}

Apesar do reconhecido envolvimento imunológico, permancem ainda por esclarecer diversos fenómenos implicados na respectiva imunopatogénese.

De acordo com a classificação de Gell e Coombs, as reacções de hipersensibilidade a fármacos podem ser classificadas em diferentes tipos, segundo os mecanismos fisiopatológicos envolvidos ${ }^{2,5}$.

Foi proposta recentemente a subdivisão das reacções de hipersensibilidade de tipo IV em 4 grupos, em função do padrão de citocinas e células efectoras envolvidas $^{2,5-7}$ : 


\section{Educação Médica Contínua}

Tabela 1 - Reacções de hipersensibilidade a fármacos - Mecanismos imunopatogénicos.

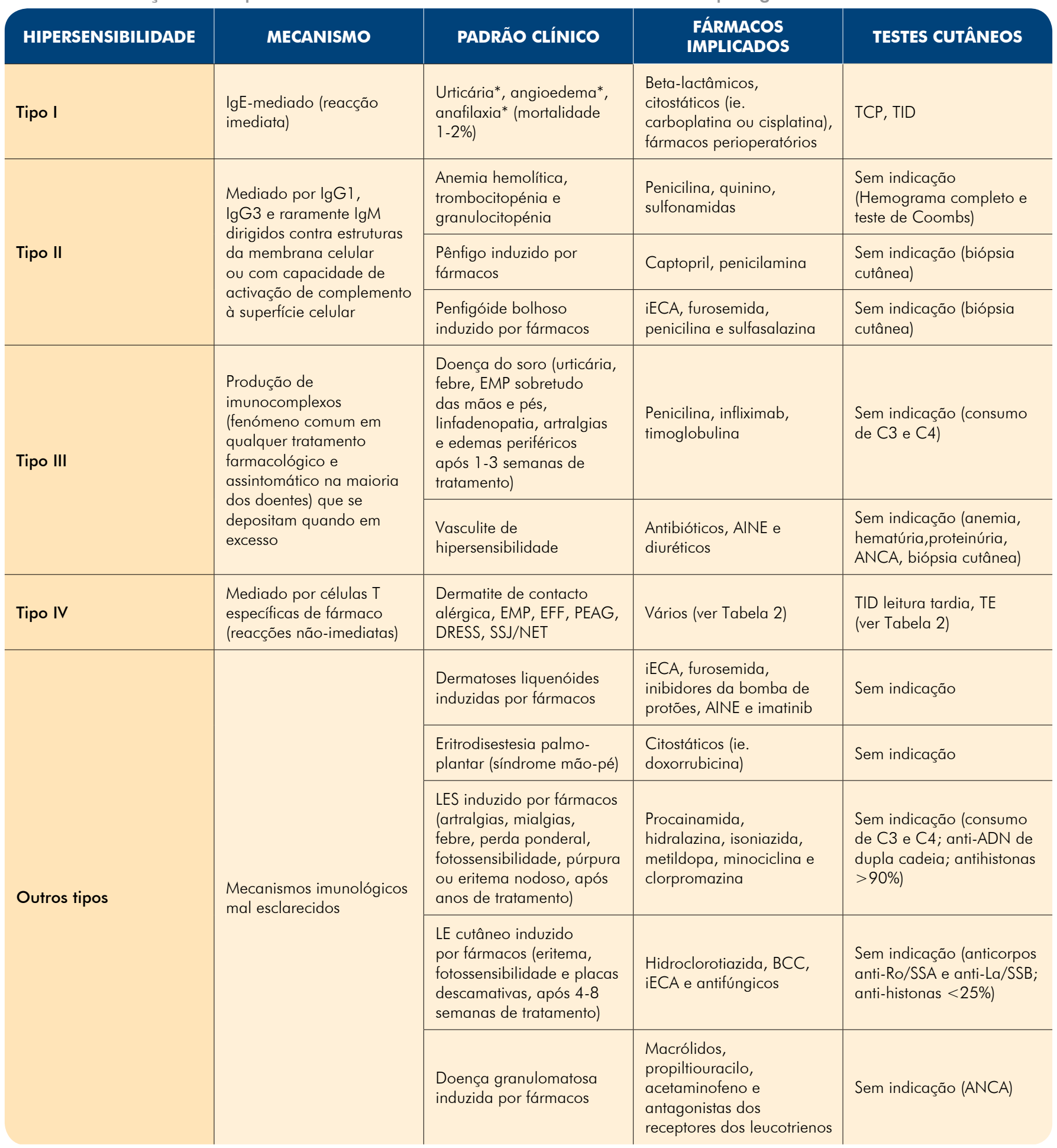

*Estas reacções podem ser não-lgE-mediadas (ie. doença do soro) ou não imunológicas (ie. associadas a AINES ou fármacos histamino-libertadores, como opióides ou relaxantes musculares). AINE, anti-inflamatórios não-esteróides; ANCA, anticorpos anti-citoplasma de neutrófilo; BCC, bloqueadores dos canais de cálcio; DRESS, síndrome de rash cutâneo com eosinofilia e sintomas sistémicos; EFF, eritema fixo a fármacos; EMP, exantemas máculo-papulares; iECA, inibidores da enzima de conversão da angiotensina; PEAG, pustulose exantemática aguda generalizada; SRIFE (Symmetrical drug-related intertriginous and flexural exanthema), exantema flexural e intertriginoso simétrico associado a fármacos; SSJ/NET, síndrome de Stevens-Johnson e necrólise epidérmica tóxica; TCP, testes cutâneos por picada; TE, testes epicutâneos; TID, testes intradérmicos. 


\section{Educação Médica Contínua}

1. IVa: monócitos e IFN-gama, sendo o eczema a manifestação clínica mais comum;

2. IVb: eosinófilos e citocinas Th2, em doentes com exantemas máculo-papulares (EMP), síndrome de hipersensibilidade a fármacos (também designado por drug rash with eosinophilia and systemic symptoms - DRESS), e síndrome de Stevens-Johnson/necrólise epidérmica tóxica (SSJ/NET);

3. IVc: células T CD4+ ou CD8+, com apoptose de queratinócitos mais ou menos extensa, nomeadamente em EMP ou SSJ/NET;

4. IVd: neutrófilos e IL-8, em doentes com pustulose exantemática aguda generalizada (PEAG).

Alguns autores adoptam a designação de síndromes mistos para reacções com envolvimento de múltiplos mecanismos imunopatológicos, tais como vasculites de hipersensibilidade, lúpus eritematoso (sistémico ou cutâneo) induzido por fármacos ou doença granulomatosa induzida por fármacos ${ }^{2,7}$.

Clinicamente, as reacções alérgicas a fármacos podem ser classificadas como $\mathrm{o}^{2,7-10}$ :

1. Reacções imediatas: até 1 hora após exposição ao fármaco, sobretudo em doentes com urticária/angioedema e/ou anafilaxia, e associado a reacções de hipersensibilidade de tipo I;

2. Reacções não-imediatas: com início mais de 1 hora após exposição ao fármaco, com manifestações clínicas diversas e associado a reacções de hipersensibilidade de tipo IV sobretudo); estas incluem reacções aceleradas (entre 1 a 72 horas) ou tardias (após as 72 horas).

\section{MECANISMOS DE ACTIVAÇÃO DO SISTEMA IMUNE}

São propostos múltiplos mecanismos moleculares de activação do sistema imunitário por acção de fármacos, nomeadamente o reconhecimento antigénico ${ }^{11}$. Este é o processo implicado nas reacções alérgicas e pode resultar do processamento por células apresentadoras de antigénio (antigen-presenting cells - APC) e consequente apresentação a células $T$ (fármacos de elevado peso molecular, ie. biológicos) ou requerer, primeiramente, a ligação covalente com proteínas transportadoras para posterior processamento do complexo fármaco-hapteno pelas $\mathrm{APC}^{12,13}$. Este modelo aplica-se à maioria dos fármacos (haptenos, moléculas de baixo peso molecular capazes de induzir resposta imunológica apenas quando ligadas a proteínas de maiores dimensões) e pode implicar o metabolismo prévio do pró-hapteno e sua conversão numa molécula mais reactiva, para posterior ligação à proteína de transporte. Noutros casos verifica-se activação directa de vias de transdução de sinal por activação de receptores (ie. imiquimod via activação de TLR-7) ${ }^{11}$.

Mais recentemente foi documentado um outro mecanismo, de interacção farmacológica com receptores imunes (conceito $\mathrm{p}-\mathrm{i}$ ), baseado na ligação não-covalente do fármaco ao receptor de células $T$, permitindo a estimulação directa de células T memória ou efectoras sem processamento por APC ou sensibilização prévia ${ }^{14,15}$. Apesar do conceito de hapteno ser - paradigma explicativo mais comum na alergia a fármacos, nenhum modelo consegue explicar isoladamente a totalidade das reacções.

\section{REACTIVIDADE CRUZADA}

A existência de reactividade cruzada ( $R C$ ) entre moléculas distintas resulta da presença de determinantes antigénicos comuns entre fármacos do mesmo grupo, justificando assim a ocorrência de reacções de hipersensibilidade imunológica com várias moléculas dentro do mesmo grupo farmacológico, independentemente de exposição prévia.

Os beta-lactâmicos (BL) são o grupo mais estudado, mais frequentemente envolvido em reacções de hipersensibilidade imunológica e que mais dificuldades coloca na prática clínica diária, merecendo por isso particular destaque ${ }^{16,17}$. A amoxicilina é actualmente ○ $\mathrm{BL}$ mais reportado (reacções tardias em $5-10 \%$ dos doentes), o que poderá ser explicado, segundo alguns autores, pela evolução dos padrões de prescrição $^{18}$.

Os BL podem ser classificados em diferentes grupos, em função da respectiva estrutura molecular e têm em comum o anel BL e adicionalmente outro anel (tiazolinidina ou di-hidrotiazina) e cadeias laterais específicas de cada fármaco:

1. Penicilinas (naturais, ie. penicilina $\mathrm{C}$ ou $\mathrm{V}$ e semi-sintéticas, ie. amoxicilina, ampicilina ou flucloxacilina): possuem estrutura bicíclia (ie. anel BL e anel de tiazolinidina) e cadeias laterais;

2. Cefalosporinas (de 1 $\stackrel{a}{,} 2^{a}$ ou $3^{\underline{a}}$ geração): apresentam, em vez de tiazolinidina, um anel de di-hidrotiazina e 2 cadeias laterais (R1 e R2);

3. Carbapenemos: com anel $\mathrm{BL}$ e anel di-hidro-pirrólico, com substituição do átomo de enxofre por carbono;

4. Monobactamos: constituídos por anel BL monocíclico, sem cadeia lateral. 


\section{Educação Médica Contínua}

A resposta imunológica pode ser desencadeada contra o anel BL ou contra determinantes antigénicos formados a partir dos anéis de tiazolinidina / di-hidrotiazina ou das cadeias laterais. Assim, fenómenos de $\mathrm{RC}$ entre $\mathrm{BL}$ poderão resultar de anticorpos dirigidos contra o próprio anel BL (implicando a evicção de todos os fármacos $\mathrm{BL}$ ), ou contra outros epitopos estruturais ${ }^{2,8}$ (Fig. 1).

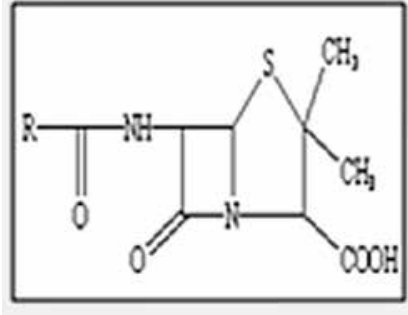

Penicilinas

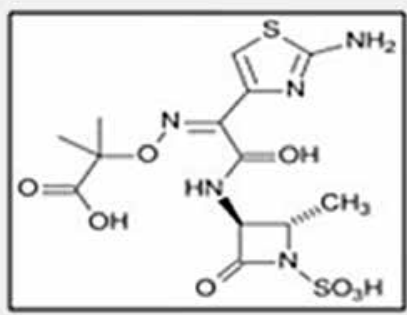

Monobactamos

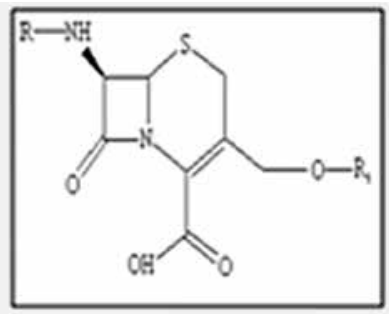

Cefalosporinas

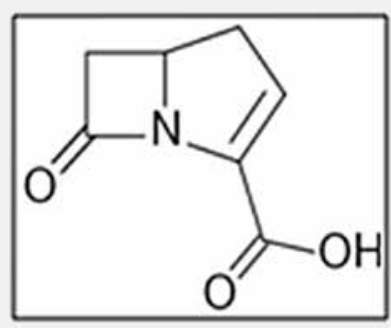

Carbapenemos
Fig 1 - Estrutura molecular de beta-lactâmicos

Atendendo às semelhanças estruturais apresentadas, admite-se a existência de RC dentro do grupo das penicilinas. No entanto, há casos descritos de alergia selectiva a amoxicilina ou ampicilina, com tolerância a outras penicilinas ${ }^{19-21}$. Este fenómeno resulta da sensibilização contra as cadeias laterais e não contra o anel BL. Assim, doentes com alergia selectiva a amoxicilina deverão evitar cefalosporinas com cadeias laterais idênticas (cefadroxil, com RC em 12-38\% dos doentes com alergia selectiva a amoxicilina, cefprozil e cefatrizina), e da mesma forma com ampicilina e cefaclor, cefalexina e cefradina ${ }^{22,23}$.

A frequência de reacções alérgicas com cefalosporinas é cerca de 10 vezes inferior à penicilina e parecem resultar maioritariamente de reacções contra as cadeias laterais ${ }^{24,25}$.

O anel BL das penicilinas é instável em condições fisiológicas, sendo rapidamente modificado por abertura espontânea. Formam-se assim haptenos que estabelecem ligações covalentes com grupos amina de outras proteínas, originando o determinante major da penicilina: benzipeniciloíl. Neste processo, o anel de tiazolinidina é geralmente preservado 8,26 . Por oposição, as cefalosporinas sofrem rápida fragmentação dos anéis $B L$ e de di-hidrotiazina, originando compostos instáveis, incapazes de funcionar como haptenos ${ }^{27,28}$. Baseado nas diferenças moleculares e metabolismo, a RC entre penicilinas e cefalosporinas associada ao anel $\mathrm{BL}$ é considerada mínima ${ }^{29}$.

Admite-se, no entanto, risco de RC entre penicilinas e cefalosporinas, sobretudo de $1^{\underline{a}}$ e $2^{\underline{a}}$ geração, em cerca de $2 \%$ dos casos, havendo mesmo casos de anafilaxia reportados ${ }^{27}$ 30-32. A RC entre cefalosporinas ou entre cefalosporinas e penicilinas resulta sobretudo de homologia das cadeias laterais (e não do anel $\mathrm{BL})^{29}$. Os monobactamos são considerados menos imunogénicos do que penicilinas ou cefalosporinas e não foi documentada RC entre penicilina e aztreonamo ou cefalosporinas e aztreonamo, à excepção da ceftazidima (cadeia lateral comum) ) $^{33,34}$. Os carbapenemos são também considerados seguros em doentes com alergia a penicilina ${ }^{35}$.

Relativamente a fenómenos de $\mathrm{RC}$ em reacções não-imediatas com $\mathrm{BL}$, os dados são escassos e pouco esclarecedores. Segundo alguns autores, as cadeias laterais parecem assumir particular relevo nas reacções mediadas por células $T$ e a RC entre cefalosporinas e penicilinas é rara ${ }^{36}$.

Relativamente ao co-trimoxazol, cerca de $3 \%$ dos doentes manifestarão reacções de hipersensibilidade mas sem RC com outras sulfonamidas, nomeadamente fármacos não-antibióticos ${ }^{37}$. A frequência de reacções imediatas com quinolonas é de $0.4-2.0 \%$ mas o grau de RC entre quinolonas de 1a, $2^{a}, 3^{\underline{a}}$ ou $4^{\mathfrak{a}}$ geração é alto, recomendando-se a evicção de todo o grupo ${ }^{38-40}$. No caso dos macrólidos, a alergia é estimada em 0.4 $3.0 \%$ dos doentes e é geralmente selectiva, com baixa RC dentro do grupo ${ }^{41}$.

\section{MANIFESTAÇÕES CLÍNICAS}

As reacções adversas a fármacos podem assumir manifestações clínicas diversas, de espectro amplo e heterogéneo e gravidade variável. Os diagnósticos diferenciais são múltiplos e a abordagem destes doentes requer por isso um elevado índice de suspeição.

Na abordagem diagnóstica destes doentes, é fundamental reconhecer o espectro de manifestações clínicas possíveis, os padrões de reacção mais característicos 


\section{Educação Médica Contínua}

para cada fármaco, sobretudo nos grupos mais frequentemente implicados, conhecer os mecanismos fisiopatológicos e as indicações e limitações dos meios de diagnóstico disponíveis.

A maioria das reacções adversas a fármacos de etiologia alérgica tem envolvimento cutâneo. Este facto poderá dever-se ao elevado número de células dendríticas e células $T$ de memória na pele, mas também às concentrações elevadas que os fármacos atingem na pele 30-45 minutos após ingestão ou por se tratar de um órgão de avaliação clínica mais acessível (nesta perspectiva, poderão ocorrer alterações de outros órgãos, nomeadamente hepáticas ou renais, transitórias e ligeiras que poderão ser subdiagnosti(adas) $)^{42,43}$.

A caracterização correcta das lesões é, por isso, fundamental para o diagnóstico e decisão terapêutica. São vários os padrões de envolvimento cutâneo, nomeadamente urticária, angioedema, EMP, eritema fixo a fármacos (EFF), PEAG, symmetrical drug-related intertriginous and flexural exanthema (SDRIFE), ou reacções mais graves, como DRESS ou SSJ/NET ${ }^{44,45}$. Além destas reacções, são também descritas reacções fotoalérgicas, dermatoses liquenóides induzidas por fármacos (nomeadamente inibidores da enzima de conversão da angiotensina, furosemida, inibidores da bomba de protões, AINE e imatinib), a eritrodisestesia palmo-plantar (síndrome mão-pé, caracterizado por edema e eritema palmo-plantar, por vezes com formação de bolhas, úlceras e necrose, geralmente 2 a 12 dias após terapêutica com citostáticos, ie. doxorrubicina), quadros de pênfigo vulgar ou penfigóide bolhoso induzidos por fármacos, vasculites de hipersensibilidade, lúpus eritematoso (sistémico ou cutâneo) induzido por fármacos, pseudolinfoma induzido por fármacos (curso indolente, com início após meses de terapêutica com anti-epiléticos, neurolépticos, iECA, beta-bloqueantes, sem febre ou outros sintomas sistémicos) $)^{44,46}$ (Tabela 2).

Os fármacos mais frequentemente implicados em reacções alérgicas cutâneas em doentes internados são amoxicilina (5.1\%), co-trimoxazol (3.4\%), ampicilina $(3.3 \%)$, sangue e derivados $(2.2 \%)$, cefalosporinas $(2.1 \%)$; eritromicina $(2.0 \%)$ e penicilina $G(1.8 \%)^{47}$.

Os EMP representam a reacção cutânea mais comum e correspondem a 31-95\% de todas as reacções

Tabela 1 - Exemplos de reacções de hipersensibilidade a fármacos não-imediatas.

\section{REACÇÃO} MECANISMO CARACTERÍSTICAS CLÍNICAS

FÁRMACOS IMPLICADOS

\begin{tabular}{|c|c|c|c|}
\hline $\begin{array}{l}\text { Exantema máculo- } \\
\text { papular }\end{array}$ & Reacção de tipo IVb ou c & $\begin{array}{l}\text { Início nas primeiras } 1 \text { a } 2 \text { semanas; descamação } \\
2 \text { a } 10 \text { dias após interrupção do fármaco; bom } \\
\text { prognóstico }\end{array}$ & $\begin{array}{l}\text { Alopurinol, aminopenicilinas, } \\
\text { cefalosporinas, trimetoprim, } \\
\text { antiepiléticos e AINE }\end{array}$ \\
\hline $\begin{array}{l}\text { Eritema fixo a } \\
\text { fármacos }\end{array}$ & $\begin{array}{l}\text { Células T CD8+ } \\
\text { intraepiteliais }\end{array}$ & $\begin{array}{l}\text { Início meses a anos após início do tratamento com } \\
\text { exacerbação } 2 \text { h após toma do fármaco; lesão única } \\
\text { nas extremidades (placa eritematosa-lívida, por vezes } \\
\text { bolha central), lábios e genitais; recorrência na mesma } \\
\text { localização em exposições subsequentes }\end{array}$ & $\begin{array}{l}\text { Sulfonamidas, tetraciclinas, } \\
\text { pirazolonas, carbamazepina, AINE }\end{array}$ \\
\hline $\begin{array}{l}\text { Pustulose } \\
\text { exantemática aguda } \\
\text { generalizada }\end{array}$ & Reacção de tipo IVd & $\begin{array}{l}\text { Início } 48 \mathrm{~h} ; \text { febre e pústulas estéreis sobretudo nas } \\
\text { pregas; neutrofilia e eosinofilia ( } 1 / 3 \text { dos casos) }\end{array}$ & $\begin{array}{l}\text { Aminopenicilinas e cefalosporinas, } \\
\text { antifúngicos, AAS, alopurinol e } \\
\text { BCC }\end{array}$ \\
\hline Síndrome de DRESS & Reacção de tipo IVb & $\begin{array}{l}\text { Início nas primeiras } 12 \text { semanas; febre, linfadenopatia, } \\
\text { hepatite aguda (50\%), pancreatite, colite, doença } \\
\text { intersticial pulmonar, envolvimento renal com nefrite } \\
\text { (10\%), enantema raro; eosinofilia (90\%); mortalidade } \\
10 \%\end{array}$ & $\begin{array}{l}\text { Antiepiléticos (raro com ácido } \\
\text { valpróico), cotrimoxazol, } \\
\text { minociclina, AINE, } \\
\text { hidroclorotiazida, nevirapina, } \\
\text { alopurinol, dapsona }\end{array}$ \\
\hline $\begin{array}{l}\text { Síndrome de Stevens- } \\
\text { Johnson / Necrólise } \\
\text { epidérmica tóxica }\end{array}$ & Reacção de tipo IVc & $\begin{array}{l}\text { Início nas primeiras } 4 \text { - } 8 \text { semanas; fase prodrómica } \\
\text { com sintomas inespecíficos (febre, toxicidade } \\
\text { sistémica); fase aguda com bolhas, descolamento } \\
\text { cutâneo (sinal de Nikolski), necrose epidérmica } \\
\text { generalizada (>30\% da superfície corporal na NET } \\
\text { ou < } 10 \% \text { no SSJ) e erosões da mucosa, leucopénia } \\
\text { e linfopénia; fase de recuperação ( } 1 \text {-3 semanas); } \\
\text { mortalidade elevada (SSJ < NET) }\end{array}$ & $\begin{array}{l}\text { Sulfonamidas ( } 1 / 3 \text { dos casos em } \\
\text { adultos), antiepiléticos, alopurinol, } \\
\text { nevirapina e AINE (nomeadamente } \\
\text { os derivados da pirazolona ou } \\
\text { oxicam, com semi-vidas mais } \\
\text { longas - maior risco no início do } \\
\text { tratamento) }\end{array}$ \\
\hline
\end{tabular}

AAS, aspirina; iECA, inibidores da enzima de conversão da angiotensina; AINE, anti-inflamatórios não-esteróides; BCC, bloqueadores dos canais de cálcio. 


\section{Educação Médica Contínua}

cutâneas induzidas por fármacos, ocorrendo em 2-8\% dos doentes internados ${ }^{48,49}$. Fazem diagnóstico diferencial com exantemas virais, sobretudo na criança (EBV, $\mathrm{CMV}$, parvovírus $\mathrm{B} 19$, enterovírus, adenovírus, HHV-6, rubéola ou sarampo), infecção por HIV ou sífilis secundária, no adulto ${ }^{50}$.

Existem porém manifestações mais raras que importa também referir. $O$ aparecimento de pústulas enquadra-se geralmente em casos de acne associados a corticóides, androgénios, lítio, fenitoína, isoniazida e sirolimus. Os quadros de PEAG cursam também com aparecimento de pústulas, em maior extensão e apresentam prevalência idêntica a SSJ/NET (ver Tabela 2) 51,52

Ao contrário das restantes reacções de hipersensibilidade a fármacos, o SDRIFE é mais frequente no sexo masculino. Caracteriza-se por eritema bem delimitado da região glútea/perineal e/ou eritema em $\mathrm{V}$ da região glútea/perigenital com envolvimento de pelo menos mais uma região intertriginosa ou prega flexural ${ }^{53}$. Este padrão específico foi no passado designado como síndrome do babuíno e a nova nomenclatura permite distingui-lo de reacções de dermatite de contacto alérgica. Cursa geralmente sem sintomas sistémicos, pode evoluir com descamação e os fármacos mais frequentemente implicados são aminopenicilinas.

Os quadros de SSJ/NET constituem as formas mais graves de hipersensibilidade a fármacos. São actualmente consideradas entidades nosológicas próximas, com um espectro de gravidade crescente, mortalidade que varia entre $1-5 \%$ e $25-70 \%$ e incidência anual de 6 e 2 casos por milhão de indivíduos, respectivamente ${ }^{54}$.

As lesões podem por vezes suscitar dificuldades diagnósticas com outras entidades, como síndrome da pele escaldada por exotoxina estafilocócica (caracteristicamente sem queixas álgicas ou enantema) ou síndrome de choque tóxico causado por Staphylococcus aureus (com descamação sobretudo palmo-plantar, febre e rápida evolução para choque).

Além dos fármacos indicados na tabela 2, há também casos descritos de SSJ/NET induzidos por BL ou mesmo por diferentes antibióticos em associação ${ }^{55}$. Em cerca de $6 \%$ dos casos não há envolvimento de fármacos, admitindo-se nestes casos provável etiologia infecciosa ${ }^{56}$. Os mecanismos etiopatogénicos que conduzem a apoptose de queratinócitos são ainda mal definidos mas parecem envolver processos imunológicos e metabólicos, em particular erros nas vias de destoxificação de xenobióticos, com acumulação de metabolitos imunogénicos ou com efeito citotóxico directo ${ }^{57}$.

\section{PAPEL DOS TESTES CUTÂNEOS NO DIAGNÓS- TICO DA ALERGIA A FÁRMACOS}

A anamnese deve incluir o início, evolução e duração dos sintomas e relação temporal com a administração dos fármacos, lista exaustiva dos fármacos utilizados pelo doente (incluindo auto-medicação), dosagem, posologia e duração dos tratamentos, exposições prévias aos vários fármacos e eventuais reacções passadas.

Apesar do papel essencial da história clínica na abordagem diagnóstica, no âmbito da alergia a fármacos são reconhecidas as suas limitações, tanto pelo número de falsos negativos, como de falsos positivos - cerca de 1/3 dos casos com diagnóstico final positivo apresentam sintomas vagos e pouco consistentes e cerca de $94 \%$ dos doentes com história clínica sugestiva têm resultados negativos na avaliação diagnóstica ${ }^{58-60}$.

Desta forma, os testes cutâneos assumem uma importância central na confirmação etiológica, permitindo demonstrar a sensibilização a fármacos, devendo ser solicitados sempre em função do contexto clínico e não como método de rastreio indiscriminado ${ }^{61,62}$.

Os testes cutâneos são fidedignos para os seguintes fármacos: penicilina, relaxantes musculares e carboplatina ${ }^{63-66}$. Para os restantes são frequentemente desconhecidos os metabolitos intermediários reactivos (imunogéneos), tornando mais complexa a interpretação dos resultados ${ }^{2,7}$.

A selecção do tipo de testes cutâneos é determinada pelo mecanismo imunopatológico subjacente à reacção adversa em causa. Assim, reacções imediatas com provável mecanismo lgE mediado podem ser estudadas por testes cutâneos por picada (TCP) ou testes intradérmicos (TID) ${ }^{2,7}$.

Os TCP podem ser realizados preferencialmente com soluções parentéricas, em concentração terapêutica ou diluída (sobretudo se reacção prévia grave ou em fármacos com propriedades histamino-libertadoras, ie. relaxantes musculares) ou mesmo com soluções orais ou outras formulações preparadas em suspensão salina $^{67}$. Constituem a primeira etapa da abordagem diagnóstica e permitem confirmar sensibilização tanto para moléculas de alto como de baixo peso molecu$\operatorname{lar}^{68}$.

Perante resultados negativos nos TCP pode avançar-se para a realização de TID, mais sensíveis mas menos específicos. Estes consistem na administração intradérmica de 0.02-0.03 mL do fármaco diluído (apenas formulação parentérica), com aumentos progressivos da concentração até resultado positivo ou até atingir concentração máxima não-irritativa ${ }^{69}$. As leituras são 


\section{Educação Médica Contínua}

realizadas aos 15-20 minutos e deve ter-se em consideração o risco de reacção sistémica com este tipo de procedimentos pelo que deverão ser realizados apenas por profissionais com experiência e em meio hospitalar. Podem ocorrer resultados falsos-negativos nas primeiras 2 semanas após episódio de anafilaxia (período refractário), pelo que se aconselha um intervalo de 4 a 6 semanas entre a resolução do quadro e realização dos testes ${ }^{70,71}$.

O valor destes testes depende dos fármacos estudados - apresentam sensibilidade e valor preditivo negativo superiores para penicilina, relaxantes musculares e enzimas mas ainda indeterminado para contrastes radiológicos, AINE ou cefalosporinas ${ }^{2,7}$.

De facto, os TID estão apenas correctamente validados para os determinantes major e minor da penicilina, com valores preditivos negativo e positivo de $99 \%$ e $50 \%$, respectivamente ${ }^{71}$.

Perante reacções não-imediatas com suspeita de provável envolvimento de células $T$ específicas de fármaco (hipersensibilidade de tipo IV), poderá ponderar-se a realização de testes epicutâneos (TE) ou TID de leitura tardia ${ }^{2,7}$.

Os TE desempenham um papel fundamental no estudo da dermatite de contacto alérgica por aplicação de fármacos tópicos, mas podem também ser realizados em doentes com EMP, PEAG, EFF (aplicados no local da lesão) ou síndrome de DRESS72-76. A sua utilização em doentes com SSJ/NET é também possível mas controversa ${ }^{72-77}$. Podem ser realizados com qualquer formulação disponível, entre 6 semanas a 6 meses após remissão dos sintomas, recomendando-se a interrupção prévia de corticóides sistémicos ou outros imunossupressores pelo menos 1 mês $^{78}$.

Os fármacos com maior número de resultados positivos são BL (nomeadamente amoxicilina), cotrimoxazol, corticóides, heparinas e anti-epiléticos mas não estão disponíveis resultados validados para a maioria dos fármacos ${ }^{78}$.

A sensibilidade destes testes varia entre 11 e $43 \%$ e os fármacos mais estudados são antibióticos (BL, clindamicina e trimetoprim), anti-hipertensores e anti-epiléticos ${ }^{72,79}$. As leituras são feitas às 48 e $96 \mathrm{~h}$ e a interpretação dos resultados requer experiência, sobretudo perante falso-positivos (ie. reacção irritativa) 2,7,78. Podem ser utilizadas concentrações crescentes do fármaco (1, 5 e 10\%) e o risco de reacção sistémica é baixo, mesmo em doentes com reacções graves (ie. $\mathrm{SSJ} / \mathrm{NET}$ ).

Resultados falsos-negativos podem dever-se a má penetração cutânea (moléculas de maiores dimensões) ou baixa concentração do fármaco. Nestas situações, pode ser considerada a realização de TID de leitura tardia (às 48h), que, segundo alguns autores, possuem sensibilidade superior aos $\mathrm{TE}^{9,80}$.

Em estudos prévios, a realização de TID de leitura tardia e TE permitiu o diagnóstico em $60-100 \%$ das reacções não-imediatas com $\mathrm{BL}^{9,18}$. Contudo, em estudos recentes, a sensibilidade de TID de leitura tardia e TE na investigação de reacções não-imediatas foi de apenas $4-9 \%$, para $B L$, corticóides, anti-epiléticos ou fármacos cardiovasculares, com maior probabilidade de resultados positivos para reacções mais gra$\operatorname{ves}^{78,79,81,82}$. A gravidade da reacção poderá contribuir para uma maior sensibildiade dos testes cutâneos, justificando assim as diferenças encontradas nos vários estudos.

\section{PROVA DE PROVOCAÇÃO}

Perante casos suspeitos de alergia a fármacos mas com resultados negativos nos testes disponíveis é possível realizar-se uma prova de provocação com o fármaco suspeito. Este procedimento pode ser requerido para esclarecimento etiológico tanto de reacções imediatas como de reacções tardias ${ }^{7}$. No entanto, pelos riscos inerentes deve ser meticulosamente ponderado caso a caso e realizado apenas em meio-hospitalar e por profissionais experientes, estando contra-indicado em reacções graves, nomeadamente síndrome de DRESS, PEAG, SSJ/NET, vasculites ou alterações hematológicas induzidas por fármacos assim como em doentes com comorbilidades graves (ie. doença renal, hepática, cardio-vascular ou outras).

Este procedimento constitui o "gold-standard" no diagnóstico de hipersensibilidade a fármacos e resultados negativos confirmam tolerância actual. De facto, num estudo de Bousquet e colaboradores, $30 \%$ dos doentes alérgicos a $\mathrm{BL}$ foi diagnosticado apenas por prova de provocação (TID falsos-negativos) ${ }^{83}$.

\section{INDUÇÃO DE TOLERÂNCIA}

Em alguns doentes com diagnóstico de alergia a fármaco e para o qual não existam alternativas seguras e igualmente eficazes, é possível proceder a indução de tolerância, permitindo assim a administração segura do fármaco requerido, sem reacções adversas. Este procedimento tem sido utilizado com sucesso em reacções lgE-mediadas (ie. antibióticos - penicilina, 


\section{Educação Médica Contínua}

cefalosporinas, macrólidos, quinolonas, vancomicina ou citostáticos - taxanos e platinas), em reacções imunológicas não-lgE-mediadas (ie. co-trimoxazol em doentes com $\mathrm{VIH}$ ) e em reacções não-imunológicas (ie. aspirina ou alopurinol), estando contra-indicada noutras reacção não-lgE-mediadas, nomeadamente em reacções graves, ie. DRESS ou SSJ/NET ${ }^{84-86}$. No caso da penicilina, os cenários clínicos mais comuns são o tratamento de sífilis durante a gravidez ou doentes com neurosífilis.

A indução de tolerância consiste na administração de doses crescentes do fármaco implicado, partindo geralmente de concentrações 10-1000 vezes inferiores às concentrações com resultados positivos em TID, até alcançar a dose terapêutica em algumas horas ou dias $^{2,7}$. Trata-se no entanto de um fenómeno transitório que deverá ser repetido a cada ciclo terapêutico requerido ${ }^{2,7}$. Estima-se a ocorrência de reacções adversas em 14-30\% dos casos, sobretudo nas fases finais ou após término do procedimento ${ }^{87-92}$. São geralmente reacções de tipo I ligeiras mas há também casos descritos de reacções não-lgE mediadas durante protocolos de indução de tolerância ${ }^{87-90}$. Este tipo de procedimento deverá ser realizado apenas em centros especializados.

\section{BIBLIOGRAFIA}

1. Guglielmi L, Guglielmi P, Demoly P. Drug hypersensitivity: epidemiology and risk factors. Curr Pharm Des. 2006; 12:3309-12.

2. Joint Task Force on Practice Parameters; American Academy of Allergy, Asthma and Immunology, American College of Allergy, Asthma and Immunology, Joint Council of Allergy, Asthma and Immunology. Drug allergy: an updated practice parameter. Ann Allergy Asthma Immunol 2010; 105(4):259-73.

3. Rawlins M, Thompson W. Mechanisms of adverse drug reactions. In: Davies D, editor. Textbook of adverse drug reactions. New York: Oxford University Press; 1991. p. 18-45.

4. Borda I, Slone D, Jick H. Assessment of adverse reactions within a drug surveillance program. JAMA. 1968; 205:645-7.

5. Pichler WJ. Delayed drug hypersensitivity reactions. Ann Intern Med. 2003;139:683-93.

6. Coombs PR, Gell PG. Classification of allergic reactions responsible for clinical hypersensitivity and disease. In: Gell RR, editor. Clinical aspects of immunology. Oxford: Oxford University Press, 1968. p.575-96.
7. Mirakian R, Ewan PW, Youlten LJ, Dugué P, Friedmann PS, English JS, et al.. BSACl guidelines for the management of drug allergy. Clin Exp Allergy. 2008; 39:43-61.

8. Levine B. Immunologic mechanisms of penicillin allergy: a haptenic model system for the study of allergic diseases of man. N Engl J Med. 1966; 275:1115-25.

9. Romano A, Blanca M, Torres MJ, Bircher A, Aberer W, Brockow K, et al.. Diagnosis of nonimmediate reactions to betalactams antibiotics. Allergy. 2004; 59:1153-60.

10. Levine B, Redmond A, Fellner M, Voss HE, Levytska V.. Penicillin allergy and the heterogeneous immune responses of man to benzylpenicillin. J Clin Invest. 1966;45:1895-906.

11. Hemmi H, Kaisho T, Takeuchi O, Sato S, Sanjo H, Hoshino K, et al. Small anti-viral compounds activate immune cells via the TLR7 MyD88-dependent signaling pathway. Nat Immunol. 2002; 3:196200.

12. Landsteiner K, Jacobs J. Studies on the sensitization of animals with simple chemical compounds. J Exp Med. 1935; 61:643-56.

13. Naisbitt DJ, Williams DP, Pirmohamed M, Kitteringham NR, Park BK. Reactive metabolites and their role in drug reactions. Curr Opin Allergy Clin Immunol. 2001; 1:317-25.

14. Schmid DA, Depta JP, Luthi M, Pichler WJ. Transfection of drug-specific T-cell receptors into hybridoma cells: tools to monitor drug interaction with T-cell receptors and evaluate cross-reactivity to related compounds. Mol Pharmacol. 2006; 70:356 -65.

15. Sieben S, Kawakubo Y, Al Masaoudi T, Merk HF, Blömeke B. Delayed-type hypersensitivity reaction to $\mathrm{p}$-phenylenediamine is mediated by two different pathways of antigen recognition by specific alpha-beta human T-cell clones. J Allergy Clin Immunol. 2002; 109:1005-11.

16. Sogn DD, Evans R, Shepherd GM, Casale TB, Condemi J, Greenberger PA, et al. Results of the National Institute of Allergy and Infectious Diseases collaborative clinical trial to test the predictive value of skin testing with major and minor penicillin derivatives in hospitalized adults. Ann Intern Med. 1992; 152:1025-32.

17. Gadde J, Spence M, Wheeler B, Adkinson NF Jr. Clinical experience with penicillin skin testing in a large inner-city STD clinic. JAMA. 1993; 270:245663.

18. Terrados S, Blanca M, Garcia J, Vega J, Torres MJ, 


\section{Educação Médica Contínua}

Carmona MJ, et al. Non-immediate reactions to beta-lactams: prevalence and role of the different penicillins. Allergy. 1995; 50:563-7.

19. Vega JM, Blanca M, Garcia JJ, Carmona MJ, Miranda A, Pérez-Estrada $M$, et al. Immediate allergic reactions to amoxicillin. Allergy. 1994; 49:317-22.

20. Blanca M, Vega JM, Garcia J, Carmona MJ, Miranda A. Allergy to penicillin with good tolerance to other penicillins: study of the incidence in subjects allergic to betalactams. Clin Exp Allergy. 1990; 20:475-81.

21. Blanca M, Perez E, Garcia J, Miranda A, Fernandez $J$, Vega JM, et al. Anaphylaxis to amoxycillin but good tolerance for benzyl penicillin: in vivo and in vitro studies of specific lgE antibodies. Allergy. 1988; 43:508-10.

22. Miranda A, Blanca M, Vega JM, Moreno F, Carmona MJ, García JJ, et al. Cross-reactivity between a penicillin and a cephalosporin with the same side chain. J Allergy Clin Immunol. 1996; 98:671-7.

23. Sastre J, Quijano LD, Novalbos A, Hernandez G, Cuesta J, de las Heras $M$, et al. Clinical cross-reactivity between amoxicillin and cephadroxil in patients allergic to amoxicillin and with good tolerance of penicillin. Allergy. 1996; 51:383-6.

24. Lin RY. A perspective on penicillin allergy. Ann Intern Med. 1992; 152:930-7.

25. Marcos Bravo C, Luna Ortiz I, Vazquez Gonzalez R. Hypersensitivity to cefuroxime with good tolerance to other beta-lactams. Allergy. 1995; 50:359-61.

26. Levine B, Redmond A. Minor haptenic determinant-specific reagins of penicillin hypersensitivity in man. Int Arch Allergy Appl Immunol. 1969; 35:445-55.

27. Romano A, Gueant-Rodriguez RM, Viola M, Pettinato R, Guéant JL. Cross-reactivity and tolerability of cephalosporins in patients with immediate hypersensitivity to penicillins. Ann Intern Med. 2004; 141:16-22.

28. Mayorga C, Torres MJ, Blanca M. Cephalosporin allergy. N Engl J Med. 2002; 346:380-1.

29. Pichichero ME. Use of selected cephalosporins in penicillin-allergic patients: a paradigm shift. Diagn Microbiol Infect Dis. 2007; 57:13-8.

30. Macy E, Mangat R, Burchette RJ. Penicillin skin testing in advance of need: multiyear follow-up in 568 test result-negative subjects exposed to oral penicillins. J Allergy Clin Immunol. 2003;111:1111-15.

31. Saxon A, Beall GN, Rohr AS, Adelman DC. Immediate hypersensitivity reactions to beta-lactam antibiotics. Ann Intern Med. 1987; 107:204-15.

32. Pichichero ME, Pichichero DM. Diagnosis of penicillin, amoxicillin, and cephalosporin allergy: reliability of examination by skin testing and oral challenge. J Pediatr. 1998; 132:137-43.

33. Adkinson NF, Saxon A, Spence MR, Swabb EA. Cross-allergenicity and immunogenicity of aztreonam. Rev Infect Dis. 1985;7(4): 613-21.

34. Adkinson NF. Immunogenicity and cross-allergenicity of aztreonam. Am J Med. 1990; 88(3):3-14.

35. Saxon A, Adelman DC, Patel A, Hajdu R, Calandra GB. Imipenem cross-reactivity with penicillin in humans. J Allergy Clin Immunol. 1988; 82:213-7.

36. Blanca $M$, Romano A, Torres MJ, Férnandez J, Mayorga C, Rodriguez J, , et al. Update on the evaluation of hypersensitivity reactions to betalactams. Allergy. 2009; 64:183-93.

37. Pradal M, Birnbaum J, Vervloet D. Allergies médicamenteuses. In: D Vervloet, A Magnan, editors. Traité d'allergologie. Paris: Médecine Sciences Flammarion; 2003: p.739-73.

38. Dávila I, Diez ML, Quirce S, Fraj J, De la hoz B, Lázaro $M$. Cross-reactivity between quinolones. Allergy. 1993; 48:388-90.

39. Arias Irigoyen J, Abengózar Muela R, García Lázaro MA, Senté Sánchez CJ. Reacción adversa por quinolonas. Estudio de reactividad cruzada. Rev Esp Alergol Inmunol Clin. 1995; 10(2):87-90.

40. Muñoz-Pereira M, Lopez Serrano C, Romualdo L, Ortega N, Barranco P, Mora C. Anaphylactic reaction by norfloxacin. Allergy. 1995;50(26):211.

41. Ben Ahmed S, Scaramuzza C, Messaad D. The accuracy of the diagnosis of suspected macrolide antibiotic hypersensitivity. Allergy. 2004; 59:11303.

42. Schaerli P, Ebert L, Willimann K, Blaser A, Roos RS, Loetscher $\mathrm{P}$, et al. A skin-selective homing mechanism for human immune surveillance T cells. J Exp Med. 2004; 199: 1265-75.

43. Hari Y, Frutig-Schnyder K, Hurni M, Yawalkar N, Zanni MP, Schnyder B, et al. T cell involvement in cutaneous drug eruptions. Clin Exp Allergy. 2001; 31:1398-408.

44. McKenna JK, Leiferman KM. Dermatologic drug reactions. Immunol Allergy Clin North Am. 2004;24:399-423.

45. Shiohara T. Fixed drug eruption: pathogenesis and diagnostic tests. Curr Opin Allergy Clin Immunol. 2009; 9:316-21.

46. Lorusso D, Di Stefano A, Carone V, Fagotti A, 


\section{Educação Médica Contínua}

Pisconti S, Scambia G. Pegylated liposomal doxorubicin-related palmar-plantar erythrodysesthesia ('hand-foot' syndrome). Ann Oncol. 2007; 18:1159-64.

47. Bigby M, Jick S, Jick H, Arndt K. Drug-induced cutaneous reactions: a report from the Boston Collaborative Drug Surveillance Program on 15,238 consecutive inpatients, 1975 to 1982. JAMA. 1986; 256:3358-63.

48. Apaydin R, Bilen N, Dokmeci S, Bayram Gurler D, Yildirim G. Drug eruptions: a study including all inpatients and outpatients at a dermatology clinic of a university hospital. J Eur Acad Dermatol Venereol. 2000; 14:518-20.

49. Bigby M. Rates of cutaneous reactions to drugs. Arch Dermatol. 2001; 137: 765-70.

50. Yawalkar N. Maculopapular Drug Eruptions. In: Pichler WJ, editor. Drug Hypersensitivity. Basel: Karger; 2007. p.242-50.

51. Mahe E, Morelon E, Lechaton S, Drappier JC, de Prost $Y$, Kreis $H$, et al. Acne in recipients of renal transplantation treated with sirolimus: clinical, microbiologic, histologic, therapeutic, and pathogenic aspects. J Am Acad Dermatol. 2006; 55:139 -42 .

52. Sidoroff A, Halevy S, Bavinck JN, Vaillant L, Roujeau JC.. Acute generalized exanthematous pustulosis (AGEP): a clinical reaction pattern. J Cutan Pathol. 2001; 28:113-9.

53. Hausermann P, Harr T, Bircher AJ. Baboon syndrome resulting from systemic drugs: is there strife between SDRIFE and allergic contact dermatitis syndrome? Contact Dermatitis. 2004; 51:297-310.

54. Roujeau JC, Stern RS. Severe adverse cutaneous reactions to drugs. N Engl J Med. 1994; 331:1272 -85 .

55. Antunes J, Lestre S, Gonçalves A, Borges A, Viseu R, Baptista I. Necrólise epidérmica tóxica - valproato de sódio e vancomicina? Rev Port Imunoalergologia. $2011 ; 19(2): 101-6$.

56. Auquier-Dunant $A$, Mockenhaupt $M$, Naldi $L$, Correia O, Schroder W, Roujeau JC; SCAR Study Group. Severe Cutaneous Adverse Reactions. Correlations between clinical patterns and causes of erythema multiforme majus, Stevens - Johnson syndrome, and toxic epidermal necrolysis: results of an international prospective study. Arch Dermatol. 2002; 138:1019-24.

57. Torres MJ, Mayorga C, Blanca M. Nonimmediate allergic reactions induced by drugs: pathogenesis and diagnostic tests. J Investig Allergol Clin
Immunol. 2009; 19:80-90.

58. Rebelo GE, Fonseca J, Araujo L, Demoly P. Drug allergy claims in children: from self-reporting to confirmed diagnosis. Clin Exp Allergy. 2008; 38:191-8.

59. Solensky R, Earl HS, Gruchalla RS. Penicillin allergy: prevalence of vague history in skin test-positive patients. Ann Allergy Asthma Immunol. 2000; 85:195-9.

60. Stember RH. Prevalence of skin test reactivity in patients with convincing, vague, and unacceptable histories of penicillin allergy. Allergy Asthma Proc. 2005; 26:59-64.

61. Gruchalla RS, Pirmohamed M. Clinical practice. Antibiotic allergy. N Engl J Med. 2006; 354:601-9.

62. Tamayo E, Alvarez FJ, Rodriguez-Ceron G, Gomez- Herreras JI, Castrodeza J. Prevalence of positive prick test to anaesthetic drugs in the surgical population. Allergy. 2006; 61:952-3.

63. Markman M, Zanotti K, Peterson G, Kulp B, Webster $K$, Belinson J. Expanded experience with an intradermal skin test to predict for the presence or absence of carboplatin hypersensitivity. J Clin Oncol. 2003; 21:4611-4.

64. Tamayo E, Rodriguez-Ceron G, Gomez-Herreras JI, Fernandez A, Castrodeza J, Alvarez FJ. Prick-test evaluation to anaesthetics in patients attending a general allergy clinic. Eur J Anaesthesiol. 2006; 23:1031-6.

65. Moneret-Vautrin DA, Kanny G. Anaphylaxis to muscle relaxants: rational for skin tests. Allerg Immunol. 2002; 34:233-40.

66. Zanotti KM, Rybicki LA, Kennedy AW, Belinson JL, Webster KD, Kulp B, et al. Carboplatin skin testing: a skin-testing protocol for predicting hypersensitivity to carboplatin chemotherapy. J Clin Oncol. 2001; 19:3126-9.

67. Brockow K, Romano A, Blanca M, Ring J, Pichler W, Demoly P. General considerations for skin test procedures in the diagnosis of drug hypersensitivity. Allergy. 2002; 57:45-51.

68. Weiss ME, Adkinson NF, Tilles SA. Diagnostic testing for drug hypersensitivity. Immunol Allergy Clin North Am. 1998; 18:731-44.

69. Empedrad R, Darter AL, Earl HS, Gruchalla RS. Nonirritating intradermal skin test concentrations for commonly prescribed antibiotics. J Allergy Clin Immunol. 2003; 112:629-30.

70. Demoly P. Anaphylactic reactions - value of skin and provocation tests. Toxicology. 2005; 209: 221-3. 


\section{Educação Médica Contínua}

71. Arroliga ME, Pien L. Penicillin allergy: considering trying penicillin again. Cleveland Clin J Med. 2003; 70:313-26.

72. Barbaud $A$, Reichert-Penetrat $S$, Trechot $P$, Jacquin-Petit MA, Ehlinger $A$, Noirez $V$, et al. The use of skin testing in the investigation of cutaneous adverse drug reactions. Br J Dermatol. 1998; 139:49-58.

73. Britschgi M, Steiner UC, Schmid S, Depta JP, Senti G, Bircher $A$, et al. T-cell involvement in drug-induced acute generalized exanthematous pustulosis. J Clin Invest. 2001; 107:1433-41.

74. Cuerda Galindo E, Goday Bujan JJ, Garcia Silva $J M$, Martínez W, Verea Hernando M, Fonseca E. Fixed drug eruption from piroxicam. J Eur Acad Dermatol Venereol. 2004; 18:586-7.

75. Antunes J, Silva R, Pacheco D, Travassos R, Filipe P. Occupational allergy to cephalosporins. Dermatol Online J. 2011;17(5):13.

76. Travassos AR, Antunes J, Almeida LS, Pacheco D, Silva R, Filipe $P$. The importance of patch tests in the differential diagnosis of adverse drug reactions. An Bras Dermatol. 2011;84(1):21-3.

77. Wolkenstein P, Chosidow O, Flechet ML, Robbiola $O$, Paul $M$, Dumé $L$, et al. Patch testing in severe cutaneous adverse drug reactions, including Stevens-Johnson syndrome and toxic epidermal necrolysis. Contact Dermatitis. 1996; 35:234-6.

78. Barbaud A, Goncalo M, Bruynzeel D, Bircher A. Guidelines for performing skin tests with drugs in the investigation of cutaneous adverse drugs reactions. Contact Dermatitis. 2001; 45:321-8.

79. Lammintausta K, Kortekangas-Savolainen O. The usefulness of skin tests to prove drug hypersensitivity. Br J Dermatol. 2005; 152:968-74.

80. Torres MJ, Sanchez-Sabate E, Alvarez J, Mayorga C, Fernández J, Padial A, et al. Skin test evaluation in nonimmediate allergic reactions to penicillins. Allergy. 2004; 59:219-24.

81. Padial A, Posadas S, Alvarez J, Torres MJ, Alvarez $\mathrm{JA}$, Mayorga $\mathrm{C}$, et al. Nonimmediate reactions to systemic corticosteroids suggest an immunological mechanism. Allergy. 2005; 60:665-70.

82. Padial A, Antúnez C, Blanca-López N, Fernández
TD, Cornejo García JA, Mayorga C, et al.. Non-immediate reactions to beta-lactams: diagnostic value of skin testing and drug provocation test. Clin Exp Allergy. 2008; 38:822-8.

83. Bousquet PJ, Pipet A, Bousquet-Rouanet L, Demoly P. Oral challenges are needed in the diagnosis of beta-lactam hypersensitivity. Clin Exp Allergy. 2008;38:185-90.

84. Stark BJ, Earl HS, Gross GN, Lumry WR, Goodman EL, Sullivan TJ. Acute and chronic desensitization of penicillin-allergic patients using oral penicillin. J Allergy Clin Immunol. 1987; 79:523-32.

85. Lee CW, Matulonis UA, Castells MC. Carboplatin hypersensitivity: a 6-h 12-step protocol effective in 35 desensitizations in patients with gynecological malignancies and mast cell/lgEmediated reactions. Gynecol Oncol. 2004; 95:370-6.

86. Feldweg AM, Lee CW, Matulonis UA, Castells M. Rapid desensitization for hypersensitivity reactions to paclitaxel and docetaxel: a new standard protocol used in 77 successful treatments. Gynecol Oncol. 2005; 96:824-9.

87. Sullivan TJ. Antigen-specific desensitization of patients allergic to penicillin. J Allergy Clin Immunol. 1982; 69: 500-8.

88. Sullivan TJ, Yecies LD, Shatz GS, Parker CW, Wedner HJ. Desensitization of patients allergic to penicillin using orally administered beta-lactam antibiotics. J Allergy Clin Immunol. 1982; 69:275-82.

89. Stark BJ, Earl HS, Gross GN, Lumry WR, Goodman EL, Sullivan TJ. Acute and chronic desensitization of penicillin-allergic patients using oral penicillin. J Allergy Clin Immunol. 1987; 79:523-32.

90. Borish L, Tamir R, Rosenwasser LJ. Intravenous desensitization to beta-lactam antibiotics. J Allergy Clin Immunol. 1987; 80:314-9.

91. Wendel GD, Stark BJ, Jamison RB, Molina RD, Sullivan TJ. Penicillin allergy and desensitization in serious infections during pregnancy. N Engl J Med. 1985; 312:1229-32.

92. Chisholm CA, Katz VL, McDonald TL, Bowes WA. Penicillin desensitization in the treatment of syphilis during pregnancy. Am J Perinatol. 1997; 14:553-4. 


\section{Educação Médica Contínua}

\section{VERIFIQUE O QUE APRENDEU}

1. Sobre as reacções adversas a fármacos, assinale a opção verdadeira:

a) Podem ser de tipo A (comuns e imprevisíveis) ou de tipo B (raras e previsíveis).

b) São mediadas pelo sistema imune (reacções alérgicas) em $>30 \%$ dos casos.

c) Opióides, vancomicina e contrastes radiológicos induzem reacções pseudoalérgicas.

d) A doença péptica induzida pela aspirina é uma reacção idiossincrática.

e) A pustulose exantemática aguda generalizada não tem envolvimento imunológico.

2. Sobre os mecanismos das reacções alérgicas a fármacos, assinale a opção verdadeira:

a) As reacções imediatas são geralmente de tipo III.

b) A doença do soro é uma reacção de tipo II.

c) O lúpus eritematoso cutâneo induzido por fármacos tem sempre anticorpos anti-histonas.

d) A eritrodisestesia palmo-plantar está geralmente associada a quinolonas.

e) As reacções de tipo IV incluem o exantema máculo-papular, DRESS, PEAG ou SSJ/NET.

3. A reactividade cruzada é frequente nos seguintes grupos farmacológicos:

a) Macrólidos.

b) Quinolonas.

c) Beta-lactâmicos.

d) Anti-inflamatórios não esteróides.

e) Opções b) e c).

4. Sobre testes cutâneos no diagnóstico de alergia a fármacos, assinale a opção verdadeira:

a) Os testes epicutâneos são úteis no diagnóstico de reacções imediatas (ie. urticária e anafilaxia).

b) Os testes intradérmicos de leitura tardia parecem ser mais sensíveis que os testes epicutâneos.

c) Os testes intradérmicos podem desencadear reacções graves.

d) No eritema fixo a fármacos, os testes epicutâneos devem ser realizados sempre no antebraço.

e) Opções b) e c).

Respostas: 1. c); 2. e); 3. e); 4. e). 